Symposia Presentations

SO1 - PROGRAM SCIENCE/

IMPLEMENTATION SCIENCE METHODS: MOVING BEYOND THE TRADITIONAL RCT

\author{
Monday, July 15, 2019 \\ 10:45 AM - 12:15 PM
}

\section{SO1.1 MUST THE EVALUATION OF COMPLEX INTERVENTIONS BE COMPLEX? LEARNING FROM THE IMPACT EVALUATION OF DREAMS}

Isolde Birdthistle*. London School of Hygiene and Tropical Medicine, London, UK

10.1136/sextrans-2019-sti.17

By nature, complex interventions - with their multiple interacting components, lengthy causal pathways and feedback loops, and 'real-world' implementation with frequent heterogeneity - are not conducive to evaluation by elegant trial design. This is particularly true in the absence of randomisation, control groups, and standardisation ('different forms in different contexts'). Are we right then, to attempt to emulate a target trial in our impact evaluation of 'DREAMS' - a large investment by PEPFAR and private sector partners in a (very) complex intervention for HIV prevention? DREAMS promotes an extensive package of interventions to address the multi-dimensional nature of HIV risk. It is being scaled across purposively chosen places and populations; it targets the most vulnerable adolescent girls and young women in priority districts of subSaharan Africa. To evaluate DREAMS' impact on HIV risk and other outcomes, we are analysing longitudinal observational data in ways that aim to mimic a randomised trial, by controlling for 'confounding by indication', so as to achieve more reliable causal inference. We will share the challenges, risks and rewards of this approach. As we grapple with an evolving intervention, revolving-door participation, time-varying confounders, and alternative causal contrasts, we face burgeoning complexity. This grows further as we seek to understand the intervention itself (what does it mean to be a DREAMS beneficiary?) and how the intervention package works; for this, we draw on process and qualitative data to elucidate the roles of mediators, mechanisms and context. There is much to learn from this large complex intervention, and we are employing emerging methods to maximise learning opportunities. There is no single or simple approach, but as complexity of the evaluation mounts, we strive to resist 'The fascination of what's difficult"* and maintain a pursuit of elegance and clarity. That too is work in progress. "WB Yeats, 1916

Disclosure No significant relationships.
S01.2 HPTN 071 (POPART): FINDINGS AND LESSONS FROM A NON-TRADITIONAL RCT

Richard Hayes*. London School of Hygiene and Tropical Medicine, London, UK

10.1136/sextrans-2019-sti.18

The HPTN 071 (PopART) study was one of four large community-randomized trials carried out in sub-Saharan Africa to determine the impact of universal testing and treatment (UTT) on HIV incidence at population level. At a time when HIV incidence globally is falling too slowly to meet UNAIDS targets, UTT has been promoted as a potential key strategy to achieve steep reductions in HIV incidence.

All four of the UTT trials have now reported their primary results. This talk will summarize the results of the HPTN 071 (PopART) study, the largest of the four trials and the last to be completed. It will explore possible explanations for the findings, and also discuss the conclusions of the study alongside the results from the other three UTT trials.

Finally, we will discuss some of the key challenges in designing and implementing a community-randomized trial of this ambition and scale, and review some of the main lessons learned during the study.

\section{SO1.3 USING MULTIPLE DATA SOURCES FOR PROGRAMME EVALUATION: INTEGRATION OF PROGRAM MONITORING DATA WITH OTHER RESEARCH STUDIES}

BM Ramesh*. University of Manitoba, Department of Community Health Sciences, Winnipeg, Canada

\subsection{6/sextrans-2019-sti.19}

Background Integration of program monitoring data with focused research studies can be a powerful approach to program evaluation and outcome assessment. This paper draws on examples from a large HIV prevention program in Karnataka, India implemented by the University of Manitoba, and funded by the Bill \& Melinda Gates Foundation.

Methods Data sources included (1) routine program data to monitor coverage (2) semi-annual assessment of behavioural outcomes using rapid, unlinked anonymous methods called Polling Booth Surveys (PBS) (3) Integrated Behavioural and Biological Surveys (IBBS) and (4) mathematical modeling of HIV transmission dynamics.

Results The program monitoring data indicated that the monthly coverage of the estimated female sex workers (FSWs) increased from $68 \%$ to $76 \%$ and the monthly clinical attendance increased from $19 \%$ to $27 \%$ over a one year period. PBS demonstrated that the condom use among FSWs in last sex with any client increased from $64 \%$ to $73 \%$ over four years. IBBS indicated that HIV prevalence among the FSWs declined from $25 \%$ at baseline to $13 \%$ at end line. The mathematical modeling which used parameters from these data sources suggested that a total of over 80,000 infections were averted by the Karnataka program. The monitoring and evaluation teams were embedded within the program, independently carrying out the design, data collection, analysis and feedback.

Discussion The embeddedness of program monitoring and evaluation enabled regular feedback to program implementation in terms of which geographies to focus, which sub-groups to prioritize etc. Special intervention packages were implemented for the young and high-volume FSWs. 
Conclusion The examples presented here used interactive processes of data use throughout the program cycle through regular feedback to program implementation pon geographies/subpopulations that are lagging behind in terms of both coverage and quality.

Disclosure No significant relationships.

\section{SO1.4 EVALUATING COMPLEX PUBLIC HEALTH ISSUE VIOLENCE: UNDERSTANDING AND MEASURING VIOLENCE AND EVALUATING VIOLENCE INTERVENTIONS - LESSONS FROM STRIVE}

Sinead Delany-Moretlwe*. Wits Reproductive Health and HIV Institute, South Africa

\section{SO2 - HIV AND SYPHILIS SELF-TESTING AND SELF-COLLECTION: EMPOWERMENT, AGENCY AND IMPLEMENTATION}

\author{
Monday, July 15, 2019 \\ 10:45 AM - 12:15 PM
}

\section{S02.1 HIV SELF-TESTING IN EASTERN AND SOUTHERN AFRICA: THE STAR PROJECT}

Maryam Shahmanesh*. University College London, Institute for Global Health, London, UK

\subsection{6/sextrans-2019-sti.20}

Background HIV testing is the first step to access both HIV treatment and prevention. While there have been tremendous efforts to close the HIV testing gap, 2.7 million people in east and southern Africa still do not know their status. Men and adolescents remain a challenge to reach. The Unitaid funded and Population Services International (PSI) led HIV Self-Testing Africa (STAR) is a five-year Initiative to catalyse the scale up of HIV self-testing (HIVST). It began with establishing the evidence base and product introduction (formation), moved to inclusion of HIVST in national plans and guidelines (early scale-up), and now optimisation of service delivery for scale-up. The results have informed WHO guidance and the development of national-level policy on HIVST. In addition, the evidence generated has transformed the testing landscape, informed estimates of the market size, and encouraged market entry among potential HIVST kit manufacturers.

Discussion In this symposium we will discuss key developments toward HIVST scale-up and the evidence generated from the STAR Initiative in six African countries. We will summarise the evidence for how HIVST has supported adolescents and men to gain knowledge of their HIV status and linked them into HIV care. We will describe the consortium plans to understand the use of this person-centred technology to link young men and women to HIV prevention, and in particular voluntary male medical circumcision and HIV PreExposure Prophylaxis. Finally, we will discuss the value that our large consortium with close relationships to national and international health policy makers brought to shaping the market and building the public health evidence. Specifically, the involvement of policy and market developments supported by
WHO; country-led research teams, supported by the London School of Hygiene and Tropical Medicine; using randomised controlled trials to evaluate rigorous interventions independently implemented by experienced country-based implementation teams (PSI).

Disclosure No significant relationships.

\section{S02.2 SYPHILIS SELF-TESTING: A NATIONWIDE PRAGMATIC STUDY AMONG MEN WHO HAVE SEX WITH MEN IN CHINA}

Cheng Wang*. Dermatology Hospital of Southern Medical University, Guangdong Center for STD Control and Prevention, Guangzhou, China

\subsection{6/sextrans-2019-sti.21}

Background Syphilis self-testing may help expand syphilis testing among men who have sex with men (MSM). China has had rapid scale up of HIV self-testing pilots, creating an opportunity for integrating syphilis self-testing. However, there is a limited literature on optimizing implementation of syphilis self-testing. We organized an online survey of MSM in China to examine syphilis self-testing experience and its determinants among MSM in China

Methods A cross-sectional online survey was conducted in 2018. Participants completed a survey instrument including socio-demographic characteristics, sexual behaviors, syphilis self-testing, and HIV self-testing history. Eligible participants were born biologically male, aged 16 or over, and engaged in anal or oral sex with a man at least once during their lifetime. Multivariable logistic regression was conducted to identify correlates of syphilis self-testing.

Results Six hundred ninety-nine MSM from 89 cities in 21 provinces in China completed the study. 361 (51.7\%) had ever tested for syphilis, of whom 174 (48.2\%) had ever used syphilis self-testing. Among 174 who had self-tested, 90 $(51.7 \%)$ reported that the self-test was their first syphilis test, $161(92.5 \%)$ reported that they undertook syphilis self-testing together with HIV self-testing. After adjusting for covariates, syphilis self-testing was correlated with disclosure of sexual orientation (aOR: 1.90, 95\%CI: 1.32-2.73), reporting two to five male sexual partners (aOR: 1.81, 95\%CI: 1.04-3.16), HIV self-testing (aOR: 39.90, 95\%CI: 17.00-93.61), and never tested for syphilis in the hospital (aOR: 2.96, 95\%CI: 1.86-4.72). Self-reported harms associated with syphilis selftesting were minimal.

Conclusions Scaling up syphilis self-testing could complement facility-based testing in China among MSM. Self-testing may increase first-time testing and has limited harms. Our findings suggest that syphilis self-testing could be integrated into HIV self-testing services.

Disclosure No significant relationships.

\section{SO2.3 HPV SELF-COLLECTION IN PERU: PROJECT HOPE}

Patricia Garcia*. Cayetano Heredia University, Unit of Epidemiology, STIs and HIV, Lima, Peru

10.1136/sextrans-2019-sti.22

In Peru, cervical cancer is the leading cancer among women, killing one woman every 5 hours. The human papillomavirus 